\title{
FOTOSSÍNTESE EM DIFERENTES GENÓTIPOS DE FEIJOEIRO
}

\author{
Luzia Pereira da Silva'; Maria Lucrecia Gerosa Ramos²; Andre Freire Cruz ${ }^{3}$; Walter Quadros Ribeiro \\ Junior'; Gustavo Rodrigues ${ }^{5}$; Sebastiao Alberto de Oliveira ${ }^{6}$; Maria Elisa Calbo ${ }^{7}$. \\ 1Universidade Federal de Pelotas, Pelotas, Rio Grande do Sul, Brasil, lufiaps@yahoo.com.br \\ 2Universidade de Brasília, Brasília, Distrito Federal, Brasil, lucreciaunb@gmail.com \\ ${ }^{3}$ Kyoto Prefectural University, Kyoto, Japão, andre@kpu.ac.jp \\ 4Embrapa Cerrados, Brasília, Distrito Federal, Brasil, walter.quadros@embrapa.br \\ 5Universidade de Brasília, Brasília, Distrito Federal, Brasil, grodrigues@gmail.com \\ •Universidade de Brasília, Brasília, Distrito Federal, Brasil, saoliveira1949@gmail.com \\ 7Universidade de Brasília, Brasília, Distrito Federal, Brasil, mcalbo@gmail.com
}

RESUMO: O objetivo do presente trabalho foi avaliar o efeito de taxa de fotossíntese (TF), condutância estomática (CE), matéria seca total de raiz e parte aérea, área foliar e clorofila a, clorofila b e clorofila total, sobre a produtividade em genótipos de feijoeiro. Foram realizados dois experimentos, um em campo (CP), no município de Cristalina - GO, e outro em casa de vegetação (CV), na Estação Experimental da Universidade de Brasília - DF, entre Agosto e Novembro de 2002. Na avaliação da TF e CE, utilizou-se o analisador portátil de gás por infravermelho. O teor de clorofila foi determinado pelo método de extração acetona $80 \%$ e leitura em espectrofotômetro. A biomassa vegetal foi determinada pelo método da secagem e pesagem. A área foliar foi determinada utilizando-se o medidor de área foliar. Os genótipos apresentaram valores diferenciados para a maioria dos parâmetros avaliados, tanto em campo como em casa de vegetação. Não houve correlação na maioria das combinações que envolveram épocas de avaliação, local de experimento (campo e casa de vegetação) e genótipos avaliados. O teor de clorofila não se correlacionou com qualquer dos parâmetros analisados. A taxa de fotossíntese na fase R7 apresentou correlação positiva $(r=0,627)$ com a produção de grão. Os demais fatores não se correlacionaram. A fase R7 foi a melhor época para avaliar o efeito da fotossíntese na produção de grãos.

PALAVRAS-CHAVE: Área foliar, Clorofila, Condutância estomática, Matéria seca, Produtividade, Taxa de fotossíntese.

\section{PHOTOSYNTHESIS IN DIFFERENT GENOTYPES OF COMMON BEAN}

ABSTRACT: The objective of this study was to evaluate the photosynthesis rates (PR), stomatal conductance $(E C)$, root and plant dry weight, leaf area, chlorophyll a and b, total chlorophyll, using different genotypes of common beans. A field experiment (FE) was conducted in Cristalina, GO, Brazil, and a greenhouse experiment (GH) was 
conducted at University of Brasilia, Brazil between August and November of 2002. A portable gas analyzer using infrared method was used in the evaluation of PR and EC. Chlorophyll concentration was determined by $80 \%$ acetone extraction method and a spectrophotometer. Plant biomass was estimated by measure the plant dry weight. Leaf area was estimated using a foliar area measurer. Most of the evaluated parameters indicated different values according to the genotypes in field and green house. The chlorophyll concentration had no correlation with other parameters. The photosynthetic rate had positive correlation $(r=0,627)$ with grain production at R7 plant stage. There were no correlations among other parameters. Plant stage R7 was the best date to evaluate the effect of photosynthesis in grain production.

KEYWORDS: Chlorophyll, Dry weight, Leaf area, Photosynthetic rate, Stomata conductance, Yield.

\section{FOTOSÍNTESIS EN DIFERENTES GENOTIPOS DE FRIJOL}

RESUMEN: El objetivo del presente trabajo fue evaluar el efecto de tasa de fotosíntesis (TF), conductividad estomática (CE), materia seca total de raíz y parte aérea, área foliar y clorofila a, clorofila be clorofila total, sobre la productividad en genotipos de frijol. Se realizaron dos experimentos, uno en campo (CP), en el municipio de Cristalina - GO, y otro en casa de vegetación (CV), en la Estación Experimental de la Universidad de Brasília - DF, entre agosto y noviembre de 2002 de la TF y CE, se utilizó el analizador portátil de gas por infrarrojo. El contenido de clorofila fue determinado por el método de extracción acetona 80\% y lectura en espectrofotómetro. La biomasa vegetal se determinó mediante el método de secado y pesaje. El área foliar se determinó utilizando el medidor de área foliar. Los genotipos presentaron valores diferenciados para la mayoría de los parámetros evaluados, tanto en campo como en casa de vegetación. No hubo correlación en la mayoría de las combinaciones que involucraron épocas de evaluación, lugar de experimento (campo y casa de vegetación) y genotipos evaluados. El contenido de clorofila no se correlacionó con ninguno de los parámetros analizados. La tasa de fotosíntesis en la fase R7 presentó correlación positiva $(r=0,627)$ con la producción de grano. Los demás factores no se correlacionaron. La fase R7 fue la mejor época para evaluar el efecto de la fotosíntesis en la producción de granos.

PALABRAS CLAVE: Área foliar, Clorofila, Conductividad estomática, Materia seca, Productividad, Tasa de fotosíntesis. 


\section{INTRODUÇÃO}

O Brasil é um país mundialmente destacado na produção e consumo de feijão (Phaseolus vulgaris L.), sendo esta leguminosa uma fonte de proteína, fibra e ferro no país (MOURA; CANNIATTI-BRAZACA, 2006; FROTA et al., 2008). Isto se deve à boa adaptação da cultura do feijoeiro às mais variadas condições edafoclimáticas existente no Brasil. Esta cultura é plantada em diferentes regiões, épocas do ano (até três safras) e sistemas de produção, que vão desde a agricultura de subsistência em pequenas propriedades à agricultura de alta tecnologia em grandes propriedades (YOKOYAMA et al., 1996).

Devido à necessidade de aumento da produtividade da cultura para melhorar sua eficiência de rendimento e consequentemente dividendo ao produtor, é importante definir os fatores que estão envolvidos na produtividade do feijoeiro. Existem diversas formas de se aumentar a produção de uma cultura como, por exemplo: abertura de novas áreas, utilização de tecnologias e melhoramento genético de cultivares (ZIMMERMANN et al., 1996). Entre esses fatores temos as características genéticas, que estão ligados à fisiologia da planta, tais como melhor eficiência fotossintética e maior aproveitamento de nutrientes fornecidos à planta.

A fotossíntese acontece, principalmente, em folhas maduras que funcionam como fonte de compostos orgânicos. Por outro lado, estas folhas fornecem o carbono para os tecidos que funcionam como dreno de compostos orgânicos, tais como raízes e frutos em desenvolvimento (WANG et al., 1999). Respostas em termos fisiológicos de genótipos de feijoeiro foram avaliadas após exposição a quantidades acumulativas de ozônio fitotóxico, onde se conclui que alta sensibilidade a este elemento foi oriunda da capacidade antioxidante da planta (VILANYI et al., 2014). De fato, o ozônio pode induzir a uma deterioração da capacidade fotossintética que está ligada a danos nas membranas sintéticas. Sintomas visíveis em $P$. vulgaris têm sido detectados através de rompimentos nas células como consequência de depósitos de $\mathrm{H}_{2} \mathrm{O}_{2}$ (GEROSA et al., 2009). 
A fotossíntese é influenciada pelas propriedades da folha e pelas práticas culturais do manejo. Estimativas do total de luz interceptadas têm sido usadas para predizer o potencial de produtividade de fruteiras (JUNG; CHOI, 2010). A distribuição da luz dentro da planta é importante para a produção, pois é lá que a energia luminosa é transformada em energia química, armazenada e transferida para os órgãos de armazenamento ou reprodução.

Estudos feitos para algumas culturas, como a soja, indicam que o aumento na produtividade ao longo dos anos foi devido à seleção de genótipos com maior capacidade fotossintética (BARONIYA et al., 2014). Fisiologicamente, uma maior absorção de nutrientes pela planta leva ao melhor aproveitamento destes aplicados na cultura, portanto genótipos mais eficientes na absorção de nutrientes e altamente produtivos são interessantes para a redução no custo de produção da cultura.

O objetivo deste trabalho foi avaliar a variabilidade genotípica na fotossíntese, condutância estomática, teor de clorofila e área foliar em diversos cultivares e linhagens de feijão, cultivados em condições de campo e casa de vegetação e correlacionar estas variáveis com a produção de biomassa e de grãos.

\section{MATERIAL E MÉTODOS}

EXPERIMENTO EM CAMPO

O trabalho foi realizado na propriedade da empresa Sementes Prezzoto Ltda, no município de Cristalina - GO, em uma área previamente utilizada como pastagem. O clima do local é do tipo tropical chuvoso de inverno seco e o solo classificado como Latossolo Vermelho-Amarelo textura argilosa, segundo classificação do Serviço Nacional de Levantamento e Classificação do Solo (Embrapa - SNLCS, RJ) e inundado no período chuvoso. A área de várzea foi sistematizada em 1999, antes do estabelecimento do experimento, e em 2000, fez-se a calagem 30 dias antes do plantio e em seguida cultivou-se feijão. Foram usados vinte genótipos de feijão: seis cultivares e quatorze linhagens, fornecidas pela empresa FT- Sementes Ltda. Algumas características agronômicas destes genótipos, obtidas em diferentes ensaios, estão descritas na Tabela 1. 
O experimento foi instalado no período de Agosto a Novembro de 2002. A análise de solo, realizada antes da instalação do experimento apresentou os seguintes atributos: $\mathrm{pH}$ em água $=, 5,2$; matéria orgânica, $=55 \mathrm{~g} \mathrm{dm}^{-3} ; \mathrm{Al}^{3+}=$, $0,03 \mathrm{cmol}_{\mathrm{c}} \mathrm{dm}^{-3} ; \mathrm{Ca}^{2+}+\mathrm{Mg}^{2+}=, 4,29$ $\mathrm{cmol}_{c} \mathrm{dm}^{-3} ; \mathrm{H}^{+}+\mathrm{Al}^{3+}=, 8,26 \mathrm{cmol}_{\mathrm{c}} \mathrm{dm}^{-3} ;$ $P=, 18,3 \mathrm{mg} \mathrm{dm}{ }^{-3} ; K={ }^{2+} 0,10 \mathrm{cmol}_{c} \mathrm{dm}^{-3}$. As correções e adubações foram feitas com base na quinta aproximação (RIBEIRO et al., 1999) e de acordo com as recomendações técnicas para a cultura do feijão da EMBRAPA (STONE; SARTORATO, 1994). Foi feita a seguinte adubação: 600 kg ha ${ }^{-1}$ de NPK (7-21-16) + FTE BR-12 (9,2\% Zn; 2,17\% B; 0,8\% Cu; 3,82\% Fe; 3,4\% Mn; 0,132\% Mo) e 200 $\mathrm{kg} \mathrm{ha-1}$ de sulfato de amônio, 22 dias após a semeadura. $\bigcirc$ delineamento experimental adotado foi de blocos ao acaso com três repetições. O tamanho das parcelas foi de $5 \mathrm{~m} \times 3 \mathrm{~m}$, com espaçamento de 0,50 m entre linhas e 0,80 entre plantas. Foram coletadas as três linhas centrais de cada parcela, descontando-se 0,5 m em cada lateral como bordadura, totalizando área útil 6 $\mathrm{m}^{2}$ por parcela.
A semeadura do feijão foi realizada em 17 de agosto de 2002. Os tratos culturais aplicados nas parcelas relacionados ao controle de plantas daninhas, pragas e doenças seguiram os mesmos protocolos utilizados na fazenda onde se desenvolveu o experimento. Tendo em vista as características topográficas e a presença de lençol freático alto, optou-se por uma irrigação sub-superficial (por capilaridade).

Para a avaliação da taxa de fotossíntese e condutância estomática foi utilizado um sistema portátil de fotossíntese (modelo LCA-4, Hoddeston, da Analytical Development Comp. (ADC) Ltda, Londres - Inglaterra). As determinações foram feitas entre 9:00 e 11:00 horas da manhã na fase vegetativa (V4), floração plena (R6) e formação de vagem (R7), respectivamente 25, 46, 58 dias após a emergência. Em cada genótipo e fase de desenvolvimento foram feitas três leituras na folha mais jovem totalmente expandida (CIAT, 1986).

Após a inserção da folha na câmara de assimilação e estabilização das leituras, o que ocorria em aproximadamente 1,5 
minutos, as três medidas eram tomadas em sequência, sem remover a folha da câmara (LONG et al., 1996).

Aos 45 dias após a semeadura da cultura, três plantas de cada parcela foram coletadas para a determinação do peso da matéria seca da parte aérea (POLANIA et al., 2012). As plantas foram cortadas rente ao solo, trazidas ao laboratório, secas em estufa a $60{ }^{\circ} \mathrm{C}$ durante 72 horas e pesadas.

A colheita para a determinação do rendimento de grãos foi realizada 90 dias após o plantio, colhendo-se todas as plantas da área útil. As plantas foram secas ao sol, trilhadas e os grãos pesados, após limpeza, com a sua umidade de grãos corrigida para 13\% (BAZGHALEH et al., 2015).

\section{EXPERIMENTO EM CASA DE VEGETAÇÃO}

Este experimento foi realizado em casa de vegetação da Estação Biológica da Universidade de Brasília (UnB), DF, em 8 de outubro de 2003 a fim de comprovar em condições mais controladas os resultados de campo, utilizando-se vasos com $4 \mathrm{~kg}$ de solo. O solo foi coletado da mesma área onde foi conduzido o experimento de campo, em outubro de 2002, ficando armazenado até a semeadura do feijão. Os genótipos usados foram os mesmos do ensaio de campo (Tabela 1). $\bigcirc$ delineamento experimental foi em blocos ao acaso, com três repetições, totalizando 60 vasos.

Em cada vaso foram aplicados $2 \mathrm{~g} \mathrm{~kg}^{-1}$ de solo de 4-30-16 (NPK) e o substrato era composto de solo e areia estéril (3:1, v/v). Seis sementes de feijão foram plantadas por vaso e uma semana após a emergência foi feito desbaste deixandose uma planta $/$ vaso $^{-1}$.

Para a avaliação da taxa de fotossíntese e condutância estomática foram utilizados os mesmos procedimentos e épocas de medição do experimento de campo na área de leitura da folha de $625 \mathrm{~mm}^{2}$.

Para a determinação dos teores de clorofila, foram coletados, de cada parcela, seis discos foliares de 5,44 mm de diâmetro de cada parcela. As amostras foram mantidas em gelo e levadas para o Laboratório de Biologia Vegetal da Embrapa Cerrados, onde foram armazenadas em freezer $\left(-18^{\circ} \mathrm{C}\right)$ 
(SANTOS et al., 2003). A extração de clorofila $a$ e clorofila $b$ foi feita com acetona 80 \% (v/v), de acordo com a metodologia e fórmula proposta por Porra et al. (1989), com algumas modificações. Os discos foliares foram macerados em almofariz de porcelana com adição acetona 80\% e o extrato centrifugado a 15.000 rpm por 5 minutos (Microcentrifuge 5415C, Eppendorf, Germany). O sobrenadante foi transferido para um balão volumétrico e o volume completado para $10 \mathrm{~mL}$. As leituras de absorbância em 646 e 663 nm foram realizadas em um espectrofotômetro de duplo feixe Shimadzu UV-190 (Shimadzu, Japão).

Após a última avaliação de fotossíntese (R7), as plantas foram retiradas do vaso, e as raízes separadas do solo e lavadas, assim como a parte aérea foi separada do sistema radicular. As folhas foram retiradas e a área foliar verde de cada planta determinada com um medidor automático de área foliar (AAC 400) (Hayashi Denkoh, Toquio, Japão). O peso da matéria seca da parte aérea (folhas, caule e vagens) e do sistema radicular foi determinado após secagem a $60{ }^{\circ} \mathrm{C}$ por 72 horas.

As análises estatísticas dos dados foram realizadas no programa Microsoft Excel associado com o SPSS 10.0. As médias foram comparadas pelo teste de Duncan, ao nível de significância de 1\% e 5\%. A correlação linear entre os parâmetros avaliados em diferentes genótipos e condições foram feitas de acordo com Gomes (1985).

\section{RESULTADOS E DISCUSSÃO}

No experimento de campo, os valores de condutância estomática apresentaram diferenças significativas entre os genótipos de feijoeiro no estádio V4 e R6. Já no estádio R7 não houve diferença significativa. No ensaio de campo, a Radiação Fotossinteticamente Ativa (RFA) variou de 477 a $1400 \cdot \cdot \mathrm{mol} \mathrm{m}^{-2} \mathrm{~s}^{-1}$ e a temperatura da folha variou de 29,7 a $35,7{ }^{\circ} \mathrm{C}$ numa área de leitura da folha de $625 \mathrm{~mm}^{2}$. A taxa de fotossíntese (TF) apresentou diferença significativa entre os genótipos de feijoeiro nas três épocas avaliadas, V4, R6 R7 (Tabela 2). 
Tabela 1. Características agronômicas dos genótipos de feijão usados no experimento.

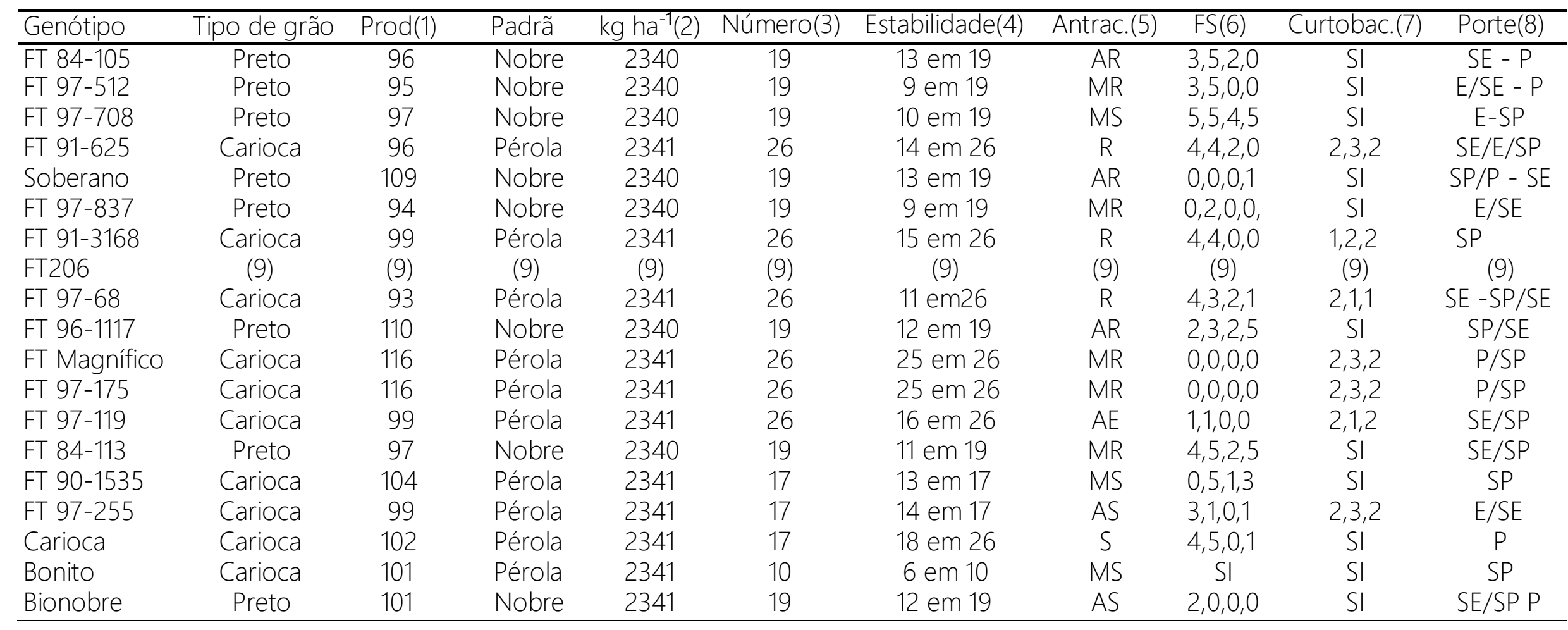

1) Produtividade relativa em relação ao padrão (\%)

2) Produtividade média em $\mathrm{kg}_{\text {ha }}{ }^{-1}$ do padrão

3) Número total de experimentos

4) Número de experimentos iguais ou superior ao padrão

5) R (resistente), AR (altamente resistente),MS (moderadamente susceptível), S (susceptível), AS (altamente susceptível)

6) Fungo de solo (fusarium e rizoctonia) - 0 a 5

7) Curtobacterium (0 a 5) - SI= sem informações

8) Porte: E (Ereto), P(Prostrado), SE-SP (semiereto ou semi prostrado) 
O peso da matéria seca da parte aérea, no ensaio de campo indicou diferença significativa entre os genótipos avaliados, assim como na produtividade dos genótipos avaliados (Tabela 2). Os genótipos que se apresentaram como sendo menos produtivos foram Carioca e Bionobre, e os mais produtivos foram FT-97-176 e FT-9768, indicando assim perspectivas para seleção destas linhagens no que diz respeito à produtividade.

No experimento de casa-devegetação, os valores de CE não apresentaram diferença significativa para o estádio V4. A RFA oscilou entre 307 a $1280 \cdot \mathrm{mol}$ fótons $\mathrm{m}^{-2} \mathrm{~s}^{-1}$ e a temperatura da folha de 25,9 a $32,9{ }^{\circ} \mathrm{C}$. Houve diferenças significativas entre os genótipos de feijoeiro no estádio R6 e R7, similarmente a TF apresentou diferença significativa entre os genótipos de feijoeiro nas três épocas avaliadas (Tabela 3). A queda da taxa de fotossíntese, no estádio R7, pode ser atribuída ao próprio estágio fisiológico da planta, no qual parte dos fotoassimilados é alocada para formação de grãos. Os valores de condutância estomática encontrados neste trabalho, no estádio V4, foram menores que o encontrado por Pimentel e Hébert (1999). Os autores encontraram para o genótipo BAT 117 de P. vulgaris, sem estresse hídrico, valores de 0,42 mol $\mathrm{m}^{-2} \mathrm{~s}^{-1}$. Por outro lado, a TF encontrada pelos autores apresentaram praticamente valores similares de TF encontrados no presente experimento.

Os valores de condutância estomática no experimento de campo nos três estádios e nos20 genótipos avaliados, em geral, foram menores que os da casa-de-vegetação. Isto mostra que no campo ocorre um estresse maior que influencia diretamente os valores de condutância estomática (Tabelas 2 e 3). Segundo Larcher (2000), o grau de abertura estomática se ajusta continuamente às oscilações dos fatores ambientais, mas o comportamento das células fechadoras depende de sua reatividade endógena. Além do mais, uma combinação de efeitos estomatais e não-estomatais influenciam na fotossíntese, dependendo da extensão do estress hídrico e às vezes quando as plantas estão bem hidratadas (YU et al., 
2009). Até mesmo níveis medios de estress hídrico podem reduzir a fotossíntese em plantas sensíveis a este fator ambiental como no caso o feijoeiro (SANTOS et al., 2009).

Os teores de clorofila a, b e total, no ensaio de casa de vegetação, apresentaram diferença significativa entre os genótipos avaliados (Tabela 3). Os valores de teores de clorofila foram similares aos encontrados por Cowling \& Sage (1998) para feijoeiro. A relação entre a clorofila a e a clorofila b em todos os genótipos foi em torno de 1,39, valores característicos de planta de sol, segundo Silva (2001), em estudo sobre diferenças varietais nas características fotossintéticas de capim-elefante (Pennisetum purpureum) e foram semelhantes aos encontrados por Guidi et al. (2000) que estudou o efeito do ozônio sobre a clorofila em 14 cultivares de feijoeiro. Estes mesmos autores verificaram também que os teores de clorofila variaram muito entre as cultivares estudadas.

O peso da matéria seca total, raiz, parte aérea e área foliar no ensaio de casa-devegetação, apresentaram diferença significativa entre os genótipos avaliados (Tabela 4).

A linhagem FT 97-176 e a cultivar FT Magnífico apesar de serem genótipos provenientes do mesmo cruzamento, sendo, portanto, linhagens irmãs, apresentaram comportamento diferente em relação aos teores de clorofila a, b e total (Tabela 3), produção de matéria seca total e área foliar (Tabela 4), mostrando uma variação entre estes genótipos.

Os valores de CE e TF obtidos no presente experimento (campo e casa de vegetação) foram semelhantes ao descrito por Pimentel (1995) e Siebeneichler et al. (1998) para Phaseolus vulgaris L. No estádio R7, formação de vagem, houve uma queda na CE e tendência de redução na TF em relação às outras épocas, em condições controladas. No experimento de campo, na maioria dos genótipos houve tendência de aumento da TF no estádio R7, fenômeno verificado por Pimentel (1995) e Siebeneichler et al. (1998), que segundo os autores pode ser devido a fatores estomáticos e nãoestomáticos ou a ação conjunta de ambos. 
Tabela 2. Condutância estomática e taxa de fotossíntese avaliados em três estádios de desenvolvimento, matéria seca da parte aérea e produtividade de genótipos de feijoeiro (experimento de campo)

\begin{tabular}{|c|c|c|c|c|c|c|c|c|}
\hline \multirow{2}{*}{ Genótipos } & \multicolumn{3}{|c|}{ Condutância estomática $\left(\mathrm{mol} \mathrm{m} \mathrm{m}^{-2} \mathrm{~s}^{-1}\right)^{\star}$} & \multicolumn{3}{|c|}{ Taxa de fotossíntese $\left(\mu \mathrm{mol} \mathrm{m}^{-2} \mathrm{~s}^{-1}\right)$} & \multirow{2}{*}{$\begin{array}{c}\text { Matéria seca } \\
\text { parte aérea } \\
\text { (q/planta) }\end{array}$} & \multirow{2}{*}{$\begin{array}{c}\text { Produtividade } \\
\mathrm{kg} \mathrm{ha}^{-1}\end{array}$} \\
\hline & V4 & R6 & R7 & V4 & R6 & R7 & & \\
\hline FT 84-105 & $0,08 a b c^{\star \star}$ & $0,08 b c$ & $0,07 a b$ & $13,95 b c$ & $15,27 b c$ & $15,50 \mathrm{ab}$ & 14,47 cde & $1846,66 \mathrm{efg}$ \\
\hline FT 97-512 & $0,10 a b c$ & 0,10ab & $0,07 a b$ & $18,71 a$ & $13,89 b c$ & $15,24 \mathrm{ab}$ & 14,80 bcd & $2962,00 \mathrm{bcd}$ \\
\hline FT 97-708 & $0,13 a$ & $0,08 b c$ & $0,08 a$ & $15,98 a b c$ & $14,62 b c$ & $17,93 a b$ & $10,23 j$ & $3892,65 a$ \\
\hline FT 91-625 & $0,11 a b$ & $0,08 b c$ & $0,07 a b$ & $15,93 \mathrm{abc}$ & $13,88 b c$ & $16,29 \mathrm{ab}$ & 12,01ghi & 2487,77 def \\
\hline Soberano & $0,10 a b c$ & $0,08 b c$ & 0,07ab & $15,45 \mathrm{abc}$ & $14,71 b c$ & $17,60 a b$ & 12,53ghi & 2942,22bcd \\
\hline FT 97-837 & 0,08abc & $0,17 a$ & 0,06ab & $15,24 a b c$ & $15,20 b c$ & $16,37 \quad a b$ & $13,05 \mathrm{fgh}$ & 2591,11de \\
\hline FT 91-3168 & $0,05 b c$ & 0,06bcd & $0,05 a b$ & $12,98 c$ & $16,02 \mathrm{bc}$ & $17,92 \quad a b$ & $16,92 a$ & $3436,74 a b c$ \\
\hline FT206 & $0,07 a b c$ & $0,05 \mathrm{~cd}$ & 0,06ab & $16,89 a b c$ & $16,63 b c$ & $18,24 a b$ & $13,43 \mathrm{efg}$ & $3513,41 a b$ \\
\hline FT 97-68 & $0,05 c$ & 0,06bcd & $0,05 a b$ & $14,51 b c$ & $13,81 b c$ & $15,20 \quad a b$ & 13,75defg & $4109,63 a$ \\
\hline FT 96-1117 & 0,08abc & $0,07 b c$ & $0,07 a b$ & 15,71abc & $21,11 a$ & $17,19 \quad a b$ & 13,25 efgh & $3024,37 \mathrm{abcd}$ \\
\hline FT Magnífico & $0,09 a b c$ & $0,07 \mathrm{bcd}$ & $0,06 a b$ & $16,02 \mathrm{abc}$ & $16,14 b c$ & $15,76 \mathrm{ab}$ & $15,13 b c$ & $3810,81 a$ \\
\hline FT 97-175 & $0,05 b c$ & $0,07 \mathrm{bcd}$ & 0,06ab & $15,39 a b c$ & $16,35 b c$ & $17,89 a b$ & 14,41cde & $4208,15 a$ \\
\hline FT 97-119 & $0,05 c$ & $0,08 b c$ & $0,03 b$ & $15,18 a b c$ & $16,10 \mathrm{bc}$ & $18,05 a b$ & $12,88 \mathrm{gh}$ & $3757,85 a b$ \\
\hline FT 84-113 & $0,06 b c$ & $0,04 d$ & 0,06ab & $14,08 \mathrm{bc}$ & $15,04 b c$ & $19,10 \quad$ a & 14,26 cdef & $3690,37 a b$ \\
\hline FT 90-1535 & 0,09abc & $0,08 b c$ & 0,06ab & $15,33 a b c$ & $12,69 c$ & $17,41 \quad a b$ & $11,48 i$ & 2636,82cde \\
\hline FT 97-255 & $0,07 a b c$ & 0,10ab & $0,08 a$ & $15,56 a b c$ & $15,27 b c$ & $13,86 \mathrm{ab}$ & 12,68ghi & $1328,37 \mathrm{~g}$ \\
\hline Carioca & 0,08abc & $0,05 \mathrm{~cd}$ & $0,04 a b$ & $15,38 a b c$ & $14,45 b c$ & $14,92 a b$ & 11,98hi & 1897,11efg \\
\hline Bonito & 0,09abc & $0,05 c d$ & 0,07ab & 17,39ab & $15,73 b c$ & $14,89 a b$ & 12,00hi & $3467,78 a b$ \\
\hline Bionobre & $0,07 a b c$ & $0,07 \mathrm{bcd}$ & 0,06ab & $16,57 a b c$ & $13,93 \mathrm{bc}$ & $13,80 \quad b$ & $15,78 a b$ & $1767,93 \mathrm{fg}$ \\
\hline CV (\%) & 18,51 & 15,12 & 24,90 & 12,02 & 13,09 & 16,01 & 5,53 & 14,34 \\
\hline
\end{tabular}

* Os valores foram transformados em log (100.x) para realizar a análise estatística, mas na tabela são apresentados os dados originais.

** Valores seguidos da mesma letra na coluna, não apresentam diferenças significativas entre si pelo teste de Duncan $(\rho<0,05)$. 
Tabela 3. Condutância estomática e taxa de fotossíntese avaliada em três estádios de desenvolvimento, teores de clorofilas de genótipos de feijoeiro (experimento de casa-de-vegetação)

\begin{tabular}{|c|c|c|c|c|c|c|c|c|c|}
\hline \multirow[b]{2}{*}{ Genótipo } & \multicolumn{3}{|c|}{ Condutância estomática $\left(\mathrm{mol} \mathrm{m} \mathrm{m}^{-2}\right)^{-1}$} & \multicolumn{3}{|c|}{ Taxa de fotossíntese $\left(\mu \mathrm{mol} \mathrm{m}^{-2} \mathrm{~s}^{-1}\right)$} & \multirow{2}{*}{$\begin{array}{l}\text { Clorofila a } \\
\left(\mu \mathrm{mol} \mathrm{m}^{-2}\right)\end{array}$} & \multirow{2}{*}{$\begin{array}{l}\text { Clorofila b } \\
\left(\mu \mathrm{mol} \mathrm{m}^{-2}\right) \\
\end{array}$} & \multirow{2}{*}{$\begin{array}{c}\text { Clorofila total } \\
\left(\mu \mathrm{mol} \mathrm{m} \mathrm{m}^{-2}\right)\end{array}$} \\
\hline & V4 & R6 & R7 & V4 & R6 & R7 & & & \\
\hline FT 84-105 & $0,28 a^{* *}$ & $0,64 a b$ & 0,09ab & $14,04 a b c$ & $15,05 b$ & $8,45 c$ & $137,81 \mathrm{n}$ & $98,80 \mathrm{~m}$ & $236,61 n$ \\
\hline FT $97-512$ & $0,22 a$ & 0,35 bcdefg & $0,06 a b$ & $13,61 a b c$ & $13,60 b$ & $11,18 a b c$ & $187,42 b$ & $134,84 b$ & $322,26 b$ \\
\hline FT 97-708 & $0,24 a$ & 0,36abcdef & 0,06ab & $13,42 \mathrm{abc}$ & $16,50 b$ & $11,55 \mathrm{abc}$ & $161,83 h$ & $116,42 \mathrm{~g}$ & $278,26 h$ \\
\hline FT 91-625 & $0,25 a$ & 0,35 bcdefg & 0,07ab & $13,42 \mathrm{abc}$ & $14,78 b$ & $10,35 a b c$ & 133,960 & $96,38 n$ & 230,340 \\
\hline Soberano & $0,29 a$ & 0,33 cdefg & 0,06ab & $12,00 \mathrm{bc}$ & $17,75 a b$ & $12,01 \mathrm{abc}$ & $150,45 k$ & $108,24 j$ & $258,69 k$ \\
\hline FT 97-837 & $0,28 a$ & $0,24 f g$ & 0,07ab & $11,40 \mathrm{c}$ & $13,35 b$ & $9,84 a b c$ & 145,221 & $104,48 k$ & 249,701 \\
\hline FT 91-3168 & $0,24 a$ & $0,67 a$ & $0,05 b$ & $13,48 a b c$ & $15,11 b$ & $12,08 a b c$ & $177,29 d$ & $127,55 c$ & $304,83 d$ \\
\hline FT206 & $0,30 a$ & 0,45abcdef & 0,09ab & $12,00 \mathrm{bc}$ & $21,34 a$ & $11,69 a b c$ & $172,95 \mathrm{e}$ & $124,42 d$ & 297,37 e \\
\hline FT 97-68 & $0,27 a$ & 0,36abcdef & 0,07ab & 12,51abc & $14,08 b$ & $11,71 a b c$ & $141,37 \mathrm{~m}$ & $101,70 \mid$ & $242,38 m$ \\
\hline FT 96-1117 & $0,26 a$ & 0,46abcde & 0,08ab & $14,57 a b$ & $12,43 b$ & $9,94 a b c$ & $152,92 j$ & $110,02 \mathrm{i}$ & $262,94 j$ \\
\hline FT Magnífico & $0,30 a$ & 0,41abcdef & 0,06ab & $13,34 a b c$ & $14,82 b$ & $10,21 a b c$ & $162,48 \mathrm{~h}$ & 117,04 & $279,52 h$ \\
\hline FT 97-175 & $0,27 a$ & $0,33 c$ defg & 0,07ab & $15,18 a$ & $12,41 \mathrm{~b}$ & 13,13ab & $169,84 f$ & $122,19 \mathrm{e}$ & $292,02 f$ \\
\hline FT 97-119 & $0,40 a$ & 0,29defg & 0,06ab & $13,62 a b c$ & $14,35 b$ & $11,68 a b c$ & $182,12 \mathrm{C}$ & $131,02 c$ & $313,15 c$ \\
\hline FT 84-113 & $0,27 a$ & 0,28 defg & 0,09ab & $12,22 \mathrm{abc}$ & $13,65 b$ & $11,78 a b c$ & $188,95 b$ & $135,93 b$ & $324,88 b$ \\
\hline FT 90-1535 & $0,36 a$ & $0,19 g$ & 0,09ab & $12,94 a b c$ & $13,20 b$ & $9,97 a b c$ & $194,64 a$ & $140,03 a$ & $334,66 a$ \\
\hline FT 97-255 & $0,42 a$ & $0,52 \mathrm{abcd}$ & $0,11 a$ & $13,18 a b c$ & $12,56 b$ & $12,10 a b c$ & $154,61 i$ & $111,23 h$ & $265,83 i$ \\
\hline Carioca & $0,28 a$ & $0,55 a b c$ & $0,05 b$ & $12,45 a b c$ & $14,68 b$ & $11,07 a b c$ & 133,960 & $96,38 n$ & 230,000 \\
\hline Bonito & $0,39 a$ & 0,42abcdef & $0,07 a b$ & $12,21 a b c$ & $12,83 b$ & $14,21 a$ & $169,82 f$ & $122,17 e$ & $219,99 p$ \\
\hline Bionobre & $0,27 a$ & 0,42abcdef & $0,11 a$ & $11,90 b c$ & $12,64 b$ & $11,51 a b c$ & $173,73 e$ & $124,99 d$ & $298,72 e$ \\
\hline CV (\%) & 10,03 & 8,94 & 21,40 & 11,99 & 19,00 & 20,34 & 0,56 & 0,57 & 0,56 \\
\hline
\end{tabular}

* Os valores foram transformados em log (100.x) para realizar a análise estatística, mas na tabela são apresentados os dados originais.
** Valores seguidos da mesma letra na coluna, nảo apresentam diferenças significativas entre si pelo teste de Duncan ( $\cdot<0,05)$. 
A análise de correlação entre os parâmetros estudados no ensaio de casade-vegetação foi estatisticamente significativa e positiva para: matéria seca total x matéria seca (raiz e parte aérea) e área foliar; matéria seca parte aérea x área foliar; clorofila a x clorofila b e clorofila total; clorofila b x clorofila total, mas significativa e negativa para: CE V4 x matéria seca (total, raiz, parte aérea) e área foliar; TF R7 x área foliar (Tabela 5).

Para o experimento de campo, a análise de correlação entre os parâmetros estudados foi positiva para: CE R7 e TF V4 em relação à produtividade e negativa para: V4 quanto à matéria seca parte aérea (Tabela 6). Já no ensaio de campo e casa de vegetação as correlações apresentaram estes resultados e positiva para: TF R6 e fotossíntese; TF R7 e condutância estomática (Tabela 7).

$\mathrm{Na}$ maioria das combinações dos parâmetros avaliados, tanto no ensaio de casa de vegetação e de campo, a correlação entre a CE e a TF, somente em dois casos os valores de $r$ foram acima de 0,50. A correlação positiva entre esses dois parâmetros tem sido demonstrada
(SIEBENEICHLER et al., 1998) no feijoeiro, onde os valores de $r$ foram acima de 0,80. Entretanto, esses trabalhos avaliam CE e TF durante um período contínuo ou estudam o efeito de algum fator sobre a CE e TF, usando concentrações ou condições em gradiente, geralmente feito somente com um genótipo, que acabam resultando em alta correlação positiva entre esses parâmetros, já que, segundo Pimentel (1995), a CE está intimamente ligada à TF. Enquanto no presente ensaio, a avaliação de CE e TF foram instantâneos e pontuais, além de envolver muitos genótipos.

A correlação negativa entre CE na fase V4 e parâmetros de produção, como matéria seca total, raiz, parte aérea, área foliar e produção de grãos, pode ser devido ao fato de que no estádio V4, os fotoassimilados são deslocados para outros usos e não para acúmulo de reserva, pois a TF não apresentou a mesma relação para o estádio V4. No ensaio de campo para o estádio R7 houve uma correlação positiva $(r=0,63)$ entre a TF e produtividade, mostrando que quanto maior for a TF, maior será a produção de grãos. 
Tabela 4. Matéria seca da parte aérea, raiz, total e área foliar de genótipos de feijoeiro (experimento de casa-de-vegetação)

\begin{tabular}{|c|c|c|c|c|}
\hline \multirow{2}{*}{ Genótipos } & \multicolumn{3}{|c|}{ Matéria seca (g/planta) } & \multirow{2}{*}{$\frac{\text { Area foliar }}{\left(\mathrm{cm}^{2}\right)}$} \\
\hline & Total & Raiz & Parte área & \\
\hline FT 84-105 & 19,03 cde & 5,30defg & $13,73 \mathrm{bcd}$ & $3.260 a$ \\
\hline FT 97-512 & $22,70 a$ & 7,49ab & $15,21 a b$ & $2.994 \mathrm{C}$ \\
\hline FT 97-708 & 18,71def & $8,07 a$ & 10,65ghi & $2.244 h$ \\
\hline FT 91-625 & $17,31 \mathrm{fgh}$ & $4,98 f g$ & 12,33 defg & $2.549 \mathrm{fg}$ \\
\hline Soberano & $15,62 \mathrm{hi}$ & $4,10 i$ & 11,52efgh & $2.437 \mathrm{~g}$ \\
\hline FT 97-837 & 19,86bcde & $6,06 \mathrm{cde}$ & $13,80 \mathrm{bc}$ & $3.060 \mathrm{~b}$ \\
\hline FT 91-3168 & $21,13 a b$ & $6,09 \mathrm{~cd}$ & $15,04 a b$ & $3.012 \mathrm{bc}$ \\
\hline FT 206 & $19,25 b c d e$ & $6,08 \mathrm{~cd}$ & $13,17 \mathrm{cde}$ & $1.839 i$ \\
\hline FT 97-68 & $20,95 a b c$ & $7,88 a$ & 13,07cde & 2.651ef \\
\hline FT 96-1117 & $17,86 \mathrm{ef}$ & $6,93 b$ & 10,93fghi & $2.497 \mathrm{fg}$ \\
\hline FT Magnífico & 18,15def & $5,07 \mathrm{fg}$ & 13,09cde & $2.260 \mathrm{~h}$ \\
\hline FT 97-175 & $16,94 \mathrm{gh}$ & $5,14 \mathrm{fg}$ & 11,79efgh & $2.856 \mathrm{~cd}$ \\
\hline FT 97-119 & 15,37hi & 5,30defg & 10,07hi & $2.477 \mathrm{fg}$ \\
\hline FT 84-113 & $20,04 \mathrm{bcd}$ & $5,78 \mathrm{cdef}$ & $14,26 a b c$ & $2.567 \mathrm{fg}$ \\
\hline FT 90-1535 & $16,70 \mathrm{~h}$ & $5,12 \mathrm{fg}$ & 11,58 efgh & $2.137 h$ \\
\hline FT 97-255 & $14,42 i$ & 4,86gh & $9,55 i$ & $1.968 \mathrm{i}$ \\
\hline Carioca & 18,03def & $5,53 \mathrm{cdefg}$ & $12,50 \mathrm{cdef}$ & $2.544 \mathrm{fg}$ \\
\hline Bonito & 15,32hi & $4,22 \mathrm{hi}$ & 11,10fghi & $1.931 i$ \\
\hline Bionobre & $20,92 a b c$ & $5,27 \mathrm{efg}$ & $15,65 a$ & $2.776 \mathrm{de}$ \\
\hline CV (\%) & 7,34 & 7,41 & 7,34 & 3,97 \\
\hline
\end{tabular}

* Valores seguidos da mesma letra na coluna, não apresentam diferenças significativas entre si pelo teste de Duncan $(\cdot<0,05)$. 
Tabela 5. Correlação linear entre os diferentes parâmetros (condutância - CE; taxa de fotossíntese - TF; matéria seca - MS; total, raiz, parte aérea (PA); área foliar; clorofila a, b, total) estudados no experimento de casa-de-vegetação

\begin{tabular}{|c|c|c|c|c|c|c|c|c|c|c|c|c|}
\hline & \multicolumn{12}{|c|}{ Parâmetros avaliados } \\
\hline & \multicolumn{3}{|c|}{ Condutância estomática } & \multicolumn{3}{|c|}{ Taxa de fotossíntese } & \multicolumn{3}{|c|}{ Matéria seca } & \multirow{2}{*}{$\begin{array}{l}\text { Área } \\
\text { foliar }\end{array}$} & \multicolumn{2}{|c|}{ Clorofila } \\
\hline & V4 & R6 & R7 & V4 & R6 & R7 & Total & Raiz & aérea & & $a$ & $b$ \\
\hline CE V4 & & & & & & & & & & & & \\
\hline CE R6 & $-0,17$ & & & & & & & & & & & \\
\hline CE R7 & $-0,17$ & 0,05 & & & & & & & & & & \\
\hline TF V4 & $-0,16$ & 0,23 & $-0,09$ & & & & & & & & & \\
\hline TF R6 & $-0,20$ & 0,19 & $-0,15$ & $-0,26$ & & & & & & & & \\
\hline TF R7 & 0,13 & 0,05 & $-0,09$ & $-0,07$ & 0,06 & & & & & & & \\
\hline MS Total & $-0,68^{* *}$ & 0,081 & $-0,09$ & $-0,15$ & 0,03 & $-0,28$ & & & & & & \\
\hline MS Raiz & $-0,51^{*}$ & $-0,04$ & $-0,23$ & 0,14 & 0,05 & $-0,19$ & $0,66^{* *}$ & & & & & \\
\hline MS PA & $-0,56^{\star *}$ & 0,13 & 0,03 & $-0,28$ & 0,01 & $-0,25$ & $0,88^{* *}$ & 0,22 & & & & \\
\hline Área foliar & $-0,51^{\star}$ & 0,11 & $-0,18$ & 0,21 & $-0,31$ & $-0,42 *$ & $0,59 * \star$ & 0,23 & $0,62 * *$ & & & \\
\hline Clorofila a & 0,17 & $-0,34$ & 0,11 & 0,025 & $-0,09$ & 0,28 & 0,13 & 0,02 & 0,16 & $-0,17$ & & \\
\hline Clorofila b & 0,17 & $-0,34$ & 0,10 & 0,023 & $-0,09$ & 0,28 & 0,13 & 0,02 & 0,16 & $-0,17$ & 1,00 ** & \\
\hline Clorofila total & $-0,02$ & $-0,34$ & 0,14 & 0,11 & $-0,01$ & 0,02 & 0,27 & 0,17 & 0,24 & 0,01 & $0,89 * *$ & $0,89 *$ \\
\hline
\end{tabular}

** Significante a $1 \%$; * significante a $5 \%$ pelo teste $t$. 
Tabela 6. Correlação linear entre os diferentes parâmetros (condutância - CE; taxa de fotossíntese - TF; matéria seca - MS; parte aérea e produtividade) estudados no experimento de campo

\begin{tabular}{|c|ccc|cr|r|}
\hline \multirow{2}{*}{} & \multicolumn{7}{|c|}{ Parâmetros } \\
\cline { 2 - 7 } CE V4 & \multicolumn{7}{|c|}{ Condutância estomática } & \multicolumn{2}{c|}{ Taxa de fotossíntese } & \\
CE R6 & \multicolumn{7}{|c|}{ R6 } & R7 & R6 & R7 & MS parte aérea \\
\cline { 2 - 7 } CE R7 & 0,29 & & & & \\
TF V4 & $0,61 * *$ & $0,45^{*}$ & & & & \\
TF R6 & $0,53^{* *}$ & 0,07 & 0,32 & & & \\
TF R7 & $-0,12$ & $-0,11$ & 0,14 & $-0,07$ & & \\
MS parte aérea & $-0,17$ & $-0,02$ & $-0,17$ & $-0,35$ & 0,29 & \\
Produtividade & $-0,40^{*}$ & $-0,27$ & $-0,29$ & $-0,22$ & 0,15 & $-0,09$ \\
\end{tabular}

** Significante a 1\%; * significante a $5 \%$ pelo teste $\mathrm{t}$. 
Tabela 7. Correlação linear entre os diferentes parâmetros (condutância - CE; taxa de fotossíntese - TF; matéria seca na parte aérea) estudados no experimento de casa-de-vegetação e campo

\begin{tabular}{|c|c|c|c|c|c|c|c|}
\hline \multirow{4}{*}{$\begin{array}{c}\text { Campo (parâmetros) } \\
\text { CE V4 }\end{array}$} & \multicolumn{7}{|c|}{ Casa-de-vegetação (parâmetros) } \\
\hline & \multicolumn{3}{|c|}{ Condutância estomática } & \multicolumn{3}{|c|}{ Taxa de fotossíntese } & \multirow{2}{*}{$\begin{array}{c}\text { Matéria seca } \\
\text { parte aérea }\end{array}$} \\
\hline & V4 & R6 & R7 & V4 & R6 & $\mathrm{R} 7$ & \\
\hline & $-0,07$ & $-0,22$ & $-0,07$ & $-0,12$ & 0,08 & $-0,36$ & $-0,02$ \\
\hline CE R6 & $-0,22$ & $-0,378$ & $-0,01$ & $-0,11$ & $-0,17$ & $-0,39 *$ & $-0,04$ \\
\hline CE R7 & $-0,07$ & $-0,01$ & 0,16 & 0,09 & $-0,11$ & $-0,28$ & $-0,09$ \\
\hline TF V4 & $-0,13$ & $-0,11$ & 0,08 & $-0,10$ & 0,06 & 0,24 & $-0,05$ \\
\hline TF R6 & 0,08 & $-0,17$ & 0,11 & $0,39 *$ & $-0,05$ & $-0,09$ & $-0,22$ \\
\hline TF R7 & $-0,36$ & $-0,39 *$ & $-0,32$ & 0,16 & 0,36 & $-0,01$ & $-0,09$ \\
\hline Matéria seca parte aérea & $-0,33$ & $0,39 *$ & 0,11 & 0,13 & $-0,11$ & 0,00 & $0,71^{\star \star *}$ \\
\hline
\end{tabular}

** Significante a 1\%; * significante a $5 \%$ pelo teste t. 
O estádio R7 é a fase de enchimento de grãos, portanto nesta fase há um intenso deslocamento de fotoassimilados para a formação de grão s (PORTES, 1996). Provavelmente, esta fase é a melhor época para a medição de TF na planta de feijoeiro. Apesar de a presente avaliação ter resultado em correlação positiva da TF com a produtividade, deve-se lembrar de que leituras pontuais feitas de maneira instantânea podem levar a erros de interpretação, pois nas outras épocas de avaliação não houve correlação de TF com parâmetros de produção. Diante dessa informação, pode-se parcialmente confiar nos resultados apresentados sobre a TF, sendo este não o único indicador para produtividade e status da planta.

Em relação à correlação positiva entre a taxa de fotossíntese e produtividade, Zelitch (1982) afirma que genótipos com alta capacidade de fixar $\mathrm{CO}_{2}$ são potencialmente capazes de aumentar a eficiência do uso de carbono, resultando em aumento do seu rendimento biológico e econômico. Este autor cita que em muitos trabalhos, onde não há uma correlação positiva entre a TF e produtividade, deve-se ao fato das avaliações de TF serem feitas de maneira instantânea, pontual, em um determinado estádio da planta, como foi o caso do presente experimento. Segundo ele, o ideal é avaliar a TF da planta toda, durante o ciclo da cultura, pois nos trabalhos onde foi feito desta maneira houve uma correlação positiva entre TF e produtividade.

Segundo Van lersel e Bugbee (2000), as razões da não correlação de TF com produção de matéria seca e produtividade pode ser devido a: a) a parte da folha onde é feita a medição de TF, não representa a folha como um todo; b) a folha escolhida para medição pode não ser representativa da planta toda; c) alterações diárias na TF; d) trocas de $\mathrm{CO}_{2}$ na folha durante o ciclo de desenvolvimento da planta; e) perda de $\mathrm{CO}_{2}$ devido à respiração de raiz e caule.

O trabalho de Baroniya et al. (2014) sobre o aumento da produtividade em soja mostrou que este dependeu da taxa de fotossíntese por unidade de área foliar. - aumento desses dois parâmetros tem sido de 0,5\% ao ano e o aumento de produtividade está correlacionado 
significativamente com $\mathrm{O}$ aumento do índice de colheita, TF e CE redução do índice de área foliar.

No caso do ensaio desenvolvido para o feijoeiro, no presente trabalho, os genótipos usados não apresentavam grandes diferenças de produtividade na média dos ensaios regionais (Tabela 1), mostrando que os potenciais genéticos destes genótipos são semelhantes, o que pode explicar a não obtenção de um maior valor de correlação de TF com produção de grãos.

\section{CONCLUSÃO}

A correlação entre os fatores analisados nas combinações que envolveram épocas de avaliação e local de experimento (CV e $(P)$, nos 20 genótipos avaliados não foi significante. Alguns parâmetros fisiológicos como o teor de clorofila não se correlacionaram com outros parâmetros analisados.

A taxa fotossintética, em R7, apresentou correlação positiva com a produção de grãos. A fase R7 caracterizase por ser a melhor para avaliar o efeito da fotossíntese.

\section{REFERÊNCIAS}

BARONIYA, S. S.; KATARIA, S.; PANDEY, G. P.; GURUPRASAD, K. N. Growth, photosynthesis and nitrogen metabolism in soybean varieties after exclusion of the UV-B and UV-A/B components of solar radiation. The Crop Journal, v. 2(6), 388397, 2014.

BAZGHALEH, N., HAMEL, C., GAN, Y., TAR'AN, B., KNIGHT, J.D. GenotypeSpecific Variation in the Structure of Root Fungal Communities is Related to Chickpea Plant Productivity. Applied and Environmental Microbiology, Washington, v. 81, n. 7, p. 2368-2377. 2015.

CIAT (Centro Internacional de Agricultura Tropical). Etapas de la planta de frigol común (Phaseolus vulgaris L.). Cali, Colombia, 34 p., 1986.

COWLING, S. A.; SAGE, R. F. Interactive effects of low atmospheric $\mathrm{CO}_{2}$ and elevated temperature on growth, photosynthesis and respiration in Phaseolus vulgaris. Plant, Cell \& Environment, London, v. 21, n. 4, p. 427435, 1998.

FROTA, K. M. G.; SOARES, R. A. M.; AREAS, J. A. G.. Composição química do feijão caupi (Vigna unguiculata L. Walp), cultivar BRS-Milênio. Ciência e Tecnologia dos Alimentos, Campinas, v. 28, n. 2, p. 470-476, 2008.

GEROSA, G.; MARZUOLI, R.; ROSSINI, M.; CINZIA PANIGADA, C.; MICHELE MERONI, M.; COLOMBO, R.; FAORO, F.; IRITI, M. A flux-based assessment of the effects of ozone on foliar injury, photosynthesis, and 
yield of bean (Phaseolus vulgaris L. CV. Borlotto Nano Lingua di Fuoco) in opentop chambers. Environmental Pollution, Amsterdam v. 157, p. 1727-1736, 2009.

GUIDI, L.; Di CAGNO, R.; SOLDATINI, G. F. Screening of bean cultivars for their response to ozone as evaluated by visible symptoms and leaf chlorophyll fuorescence. Environmental Pollution, Amsterdam, v.107, p. 349-355, 2000.

GOMES, F. P. Curso de estatística experimental 11 ed. Piracicaba: Livraria Nobel, 1985. 460 p.

JUNG, S.; CHOI, H. Light penetration, growth, and fruit productivity in 'Fuji' apple trees trained to four growing systems, Scientia Horticulturae, v. 125, n.4, p. 672678, 2010.

LARCHER, W. Ecofisiologia vegetal. São Carlos: RiMA, 2000. 531 p.

LONG, S. P.; FARAGE, P.K.; GARCIA, R.L. Measurement of leaf and canopy photosynthetic $\mathrm{CO}_{2}$ exchange in the field. Journal of Experimental Botany, v. 47: 1629 - 1642, 1996.

MOURA, N. C.; CANNIATTI-BRAZACA, S. G. Avaliação da disponibilidade de ferro de feijão comum (Phaseolus vulgaris L.) em comparação com carne bovina. Ciência Tecnologia dos Alimentos, Campinas, v. 26, n. 2, p. 270-276, 2006.

PIMENTEL, C. Metabolismo de carbono na agricultura tropical. Seropédica: Edur, 1995. 150 p.

PIMENTEL, C.; HÉRBERT, G. Potencial fotossintético e condutância estomática em espécies de feijão caupi sob deficiência hídrica. Revista Brasileira de Fisiologia Vegetal, Brasília, v. 11, n. 1, p. 711, 1999.

POLANÍA, J. A.; RIVERA, M.; RICAURTE, J.; RAO, I. M. Phenotyping Common Beans for Adaptation to Drought: Protocol for Greenhouse Evaluation. International Center for Tropical Agriculture.

https://cgspace.cgiar.org/bitstream/handle /10568/38748/greenhouse_evaluation_final .pdf?sequence=3. 2012. (Accessed in January 2016)

PORRA, R. J.; THOMPSON, W. A.; KRIEDEMANN, P. E. Determination of accurate extinction coefficients and simultaneous equations for assaying chlorophylls $a$ and $b$ extracted with four different solvents: verification of the concentration of chlorophyll standards by atomic absorption spectroscopy. Biochimica et Biophysica Acta, Amsterdam, v. 975, p. 384-394, 1989.

PORTES, T. A. Ecofisiologia In: ARAUJO, R.S.; RAVA, C.A.; STONE, L.F.; ZIMMERMANN, M. J. O. (Eds.).Cultura do Feijoeiro Comum no Brasil. Piracicaba: Potafos, 1996. p.101-137.

RIBEIRO, A. C.; GUIMARÃES, P. T. G. Alvarez $\vee$., $\vee$. H. Recomendações para o uso de corretivos e fertilizantes em Minas Gerais - $5^{a}$ Aproximação. SBCS (Eds). 359p. 1999.

SANTOS, A. C. A.; CALIJURI, M. C:; MORAES, E. M.; ADORNO, M. A. T.; FALCO, P. B.; CARVALHO, D. P.; DEBERDT, G. L. B.; BENASSI, S. F. Comparison of three methods for Chlorophyll determination: Spectrophotometry and Fluorimetry in samples containing pigment 
mixtures and spectrophotometry in samples with separate pigments through High Performance liquid chromatography. Acta Limnologica Brasilica, v. 15, n. 3, p 718, 2003.

SANTOS, M. G.; RIBEIRO, R. V.; MACHADO, E. C.; PIMENTEL, C. Photosynthetic parameters and leaf water potential of five common bean genotypes under mild water deficit. Biologia Plantarum, v. 53, n. 2, p. 229-236. 2009.

SIEBENEICHLER， S. C.; SANT'ANNA， R.; MARTINEZ, C. A.; MOSQUIM, P. R.; CAMBRAIA, J. Alterações na fotossíntese, condutância estomática e eficiência fotoquímica induzidas por baixa temperatura em feijoeiros. Revista Brasileira de Fisiologia Vegetal, Londrina, v. 10, n. 1, p. 37-44, 1998.

SILVA, M. M. P.; VASQUES, H. M.; BRESSAN-SMITH, R. E.; SILVA, J. F. C.; ERBESDOBLER, E. D. Diferenças varietais nas características fotossintéticas de Pennisetum purpureum Schum. Revista Brasileira de Zootecnia, Viçosa, v. 30, p. 1975-1983, 2001.

STONE, L.F.; SATORATO, A. O cultivo do feijão: recomendações técnicas. Brasília, Embrapa-SPI, 1994. 83 p. (Embrapa-CNPAF, Documentos 48).

Van IERSEL, M.W.; BUGBEE, B. A multiple chamber, semicontinuos, crop carbon dioxide exchange system: design, calibration, and data interpretation. Journal of American Society for Horticultural Science, Alexandria, v. 125, p. 86-96, 2000.
VILLÁNYI, V.; ÜRMÖS, Z.; TURK, B;. BATIČ, F.; CSINTALAN, Z. Photosynthesis of ozone-sensitive and -resistant Phaseolus vulgaris genotypes under ambient ozone and moderate heat stress. Photosynthetica, v. 52, n. 4, p. 604-613, 2014.

WANG, Z.; PAN, P.; QUEBEDEAUX, B. Carbon partitioning into sorbitol, sucrose, and starch in source and sink apple leaves as affected by elevated $\mathrm{CO}_{2}$. Environmental and Experimental Botany, Oxford, v. 41, p. 39-46, 1999.

YOKOYAMA, L. P.; BANNO, K; KLUTHCOUSKI, J. Aspectos socioeconômicos da cultura. In: ARAUJO, R.S.; RAVA, C.A.; STONE, L.F.; ZIMMERMANN, M.J.O. (Eds.). Cultura do Feijoeiro Comum no Brasil. Piracicaba: Potafos, 1996. p. 1-20.

YU, D. J., KIM, S. J., LEE, H. J. Stomatal and non-stomatal limitations to photosynthesis in field-grown grapevine cultivars. Biologia Plantarum v. 53, p. 133-137, 2009.

ZELITCH, I. The close relationship between net photosynthesis and crop yield. BioScience, Arlington, v. 32, p. $796-$ 802, 1982.

ZIMMERMANN, M. J. O.; CARNEIRO, J. E. S.; PELOSO. M. J. D.; COSTA, J. G. C.; RAVA, C. A.; SARTORATO, A.; PEREIRA, P. A. A. Melhoramento Genético e Cultivares. In: ARAUJO, R.S.; RAVA, C.A.; STONE, L.F.; ZIMMERMANN, M.J.O. (Eds.). Cultura do Feijoeiro Comum no Brasil. Piracicaba: Potafos, 1996. p. 223227 